\title{
Efeitos do Tenoxicam sobre a cicatrização da parede abdominal: estudo experimental em ratos $^{1}$
}

\author{
Effects of Tenoxicam on the abdominal wall healing: experimental study in rats
}

\author{
André Luís Conde Watanabe², Luís Massaro Watanabe ${ }^{3}$ \\ 1. Trabalho realizado no Laboratório de Cirurgia Experimental da Faculdade de Medicina da Universidade de Brasília. \\ 2. Acadêmico de Medicina da Universidade de Brasília; Bolsista de Iniciação Científica do CNPq. \\ 3. Professor Adjunto da Área de Clínica Cirúrgica da Faculdade de Medicina da Universidade de Brasília.
}

\section{RESUMO}

Objetivo: Analisar os efeitos do tenoxicam, um antiinflamatório não hormonal, na cicatrização da parede abdominal de ratos. Métodos: Foram utilizados 40 ratos adultos, submetidos à laparotomia mediana e distribuídos, aleatoriamente, em um grupo controle (C), constituído de 20 animais que receberam solução de $\mathrm{NaCl}$ a 0,9 \%; e um grupo tratado (T), constituído de 20 animais que receberam o tenoxicam. Os animais de cada grupo foram divididos, conforme a data de sacrifício, em subgrupos de 10 animais, denominados C7, C14, T7 e T14. As inscrições 7 e 14 determinaram o sacrifício dos animais no sétimo e décimo quarto dia pós-operatório, respectivamente. As soluções de tenoxicam ( $1 \mathrm{mg} / \mathrm{ml}$ ) e de $\mathrm{NaCl}$ a $0,9 \%$ foram administradas no pós-operatório imediato e nos quatro dias seguintes, por via intramuscular, na dose volume de $0,6 \mathrm{ml} / \mathrm{kg} /$ dia. No dia do sacrifício, realizou-se a ressecção de dois fragmentos da parede abdominal (1cm x 3cm), que foram utilizados para determinação da concentração de hidroxiprolina e avaliação da força de ruptura. Resultados: Não foram observadas complicações da ferida operatória, incluindo infecção ou deiscência, nos quatro subgrupos de animais. Na análise comparativa dos quatro subgrupos de animais, não foi evidenciada diferença estatisticamente significante no estudo da força de ruptura $(p=0,262)$ e na concentração de hidroxiprolina ( $p=0,392)$. Conclusão: A administração de tenoxicam, por via intramuscular, não interfere na cicatrização da parede abdominal de ratos.

Descritores: Tenoxicam. Cicatrização. Parede abdominal.

\begin{abstract}
Purpose: To analyse the effect of tenoxicam, a nonsteroidal anti-inflammatory, on the abdominal wall healing in rats. Methods: It was used 40 rats, submitted to longitudinal laparotomies, and allocated, randomly, in one control group (C), consisted of 20 rats treated with saline solution; and one test group (T), consisted of 20 rats treated with tenoxicam. The animals of each group were divided, according to their sacrifice day, into subgroups of 10 animals, named as C7, C14, T7 and T14. The numbers 7 and 14 indicated that the animal would be sacrificed on the $7^{\text {th }}$ and $14^{\text {th }}$ postoperative day, respectively. The tenoxicam $(1 \mathrm{mg} / \mathrm{ml})$ and saline solution $(\mathrm{NaCl} 0,9 \%)$ were administrated by intramuscular injections, at the dose of $0,6 \mathrm{ml} / \mathrm{kg} /$ day, immediately after surgery and continued for 4 days. In the sacrifice day, two segments of the abdominal wall $(1 \mathrm{~cm} \times 3 \mathrm{~cm})$ were prepared and submitted to breaking strength measurement and hydroxyproline determination. Results: No complications were observed in the four subgroups, including infection or dehiscence. There were no significant differences in the breaking strength measurement $(p=0,262)$ and the hydroxyproline levels $(p=0,392)$ among the four subgroups. Conclusion: The tenoxicam, administered intramuscularly, does not interfere on the abdominal wall healing of rats.
\end{abstract}

Key words: Tenoxicam. Wound healing. Abdominal wall.

\section{Introdução}

Nos procedimentos cirúrgicos abdominais, a deiscência da ferida da parede abdominal é uma complicação freqüente, com significativa taxa de morbidade pós-operatória ${ }^{1}$. Apesar dos progressos obtidos nas técnicas cirúrgicas, no material de sutura, nos cuidados pré-operatórios e pós-operatórios, a incidência de deiscência da ferida da parede abdominal varia de 0,2 a $11 \%{ }^{2,3}$. Diversos fatores locais e sistêmicos foram relacionados ao desenvolvimento dessa complicação pós-operatória. Em relação ao uso de medicamentos, somente as drogas imunossupressoras quimioterápicas ${ }^{4}$ e a terapia prolongada com corticóides ${ }^{2}$ foram consideradas fatores predisponentes.

Os antiinflamatórios não hormonais tem sido comumente utilizados para o controle da dor no período pós-operatório, principalmente porque não resultam em sedação ou em depressão respiratória. O tenoxicam é um antiinflamatório não hormonal do grupo oxicam, inibidor não seletivo da ciclooxigenase e com uma conveniente meiavida longa ${ }^{5}$. Esse fármaco revelou-se efetivo no controle da dor no pós-operatório de cirurgias abdominais ${ }^{6,7}$, sendo 
muito utilizado na prática clínica. O tenoxicam age inibindo a síntese de prostaglandinas e eicosanóides, que são fundamentais na resposta inflamatória ${ }^{8}$. Entretanto, uma das preocupações do uso dos antiinflamatórios não hormonais no perioperatório é o risco de retardar o processo de cicatrização, devido às alterações na resposta inflamatória ${ }^{9}$. Os efeitos dos antiinflamatórios não hormonais sobre a cicatrização de feridas cirúrgicas têm sido objeto de estudos experimentais, porém os resultados dessas investigações permanecem controversos. Tognini e col. ${ }^{10}$ avaliaram o efeito do diclofenaco de sódio na cicatrização da camada músculo aponeurótica da parede abdominal de ratos, utilizando-se de estudo histopatológico e análise quantitativa morfométrica de colágeno. Os autores concluíram que a cicatriz da parede abdominal dos ratos do grupo tratado continha significativamente menor quantidade de fibras de colágeno no $7^{\circ}$ e $14^{\circ}$ dias de pós-operatório, quando comparada a dos animais do grupo controle. Minossi e col. ${ }^{11}$ utilizaram a força de ruptura, o exame histopatológico e a relação hidroxiprolina/proteína tecidual como parâmetros para avaliar os efeitos do diclofenaco sódico sobre a cicatrização da parede abdominal de ratos. Os animais do grupo tratado apresentaram retardo do processo cicatricial em relação aos seus controles, caracterizado por uma menor fibrogênese, menor densidade de fibras colágenas, além de um número maior de complicações, como microabscessos, em torno dos fios de sutura. No entanto, Tognini e col. ${ }^{12}$ observaram que o meloxicam, um antiinflamatório não hormonal inibidor levemente seletivo da ciclooxigenase 2, não produz alterações biomecânicas e morfológicas na cicatrização da parede abdominal de ratos. A p ó s revisão extensa da literatura, não foram encontrados trabalhos avaliando as influências do tenoxicam na cicatrização da parede abdominal. Portanto, o presente estudo tem como objetivo avaliar os efeitos do tenoxicam sobre a cicatrização da parede abdominal em ratos.

\section{Métodos}

Foram utilizados 40 ratos machos, Rattus norvegicus, linhagem Wistar, com peso corporal entre 380 e 500 gramas e, aproximadamente, 150 dias de vida. Todos os ratos eram sadios e foram mantidos no laboratório com ração apropriada e água, à vontade. Os animais foram distribuídos aleatoriamente em dois grupos:

Grupo C - grupo controle, constituído de 20 animais que receberam solução de cloreto de sódio a 0,9\%, administrada por injeção intramuscular.

Grupo T - grupo tratado, constituído de 20 animais que receberam o tenoxicam, administrado por injeção intramuscular.

Os animais de cada grupo foram divididos em subgrupos de 10 animais, denominados C7, C14, T7 e T14. As inscrições 7 e 14 determinaram o sacrifício dos animais no sétimo e décimo quarto dia pós-operatório, respectivamente. Para o procedimento cirúrgico, os animais foram anestesiados com injeção intramuscular de solução composta por $25 \mathrm{mg} / \mathrm{Kg}$ de cloridrato de cetamina e $5 \mathrm{mg} /$ Kg de cloridrato de xilazina ${ }^{13}$. Após indução anestésica, os animais foram imobilizados em decúbito dorsal e, em seguida, realizada a tricotomia da parede abdominal e anti- sepsia com álcool iodado a $2 \%$. Realizou-se laparotomia mediana, com incisão de $3 \mathrm{~cm}$ de comprimento a partir do apêndice xifóide. A seguir, foi realizada a síntese da parede abdominal em dois planos, por meio de suturas do tipo chuleio simples contínua. No primeiro plano, procedeu-se à inclusão do músculo e da aponeurose, utilizando-se de fio de polipropilene 4-0. No segundo plano, foram incluídos o tecido celular subcutâneo e a pele, utilizando-se de fio de nylon monofilamentar 4-0. Os animais do grupo T receberam o antiinflamatório tenoxicam $(1 \mathrm{mg} / \mathrm{ml})$, na dose volume de $0,6 \mathrm{ml} / \mathrm{kg} /$ dia, administrado por injeção intramuscular, em dose única, durante cinco dias. Os animais do grupo $\mathrm{C}$ receberam a solução de cloreto de sódio a $0,9 \%$, por injeção intramuscular, na dose volume de $0,6 \mathrm{ml} / \mathrm{kg} / \mathrm{dia}$, de modo semelhante aos animais do grupo T. As administrações da solução de tenoxicam e da solução de cloreto de sódio a 0,9\% foram iniciadas no pós-operatório imediato e continuaram nos quatro dias seguintes. No dia indicado para o sacrifício, os animais foram anestesiados e, em seguida, realizada a ressecção de um fragmento quadrangular da parede abdominal, com $3 \mathrm{~cm}$ de largura e 3 cm de comprimento, contendo a sutura na sua porção central. Desse fragmento quadrangular, foram retirados dois segmentos iguais de $1 \mathrm{~cm}$ de largura por $3 \mathrm{~cm}$ de comprimento. $\mathrm{O}$ fragmento do lado do apêndice xifóide foi imerso em solução de cloreto de sódio a 0,9\% e congelado a $-20^{\circ} \mathrm{C}$, para estudo posterior da concentração de hidroxiprolina, enquanto o fragmento localizado na porção central da parede abdominal foi destinado para o estudo da força de ruptura. O processo de extração da hidroxiprolina foi realizado pela técnica proposta por Bergman e Loxley ${ }^{14} \mathrm{e}$ a dosagen foi efetuada pela técnica de Stegemann e Stalder ${ }^{15}$. O estudo da força de ruptura foi realizado em máquina de ensaios de acionamento eletrônico conectada à microcomputador (Mecmesin $®$,Versa Test, United Kingdon) imediatamente após a ressecção do fragmento. A velocidade utilizada no teste foi de $50 \mathrm{~mm}$ por minuto e os resultados foram expressos em Newton $(N)$. Durante a determinação da força de ruptura, foram dispensados cuidados rigorosos na fixação e no posicionamento dos fragmentos teciduais no aparelho de ensaio vertical, com padronização da distância entre a cicatriz cirúrgica e as pinças de fixação. Para análise estatística dos resultados, foram utilizados os testes de análise de variância e de análise de variância por postos de Kruskal-Wallis ${ }^{16}$. O nível de significância adotado em todos os testes foi de $5 \%(\mathrm{p}<0,05)$.

\section{Resultados}

Não houve diferença estatística entre os pesos dos animais dos quatro subgrupos $(p=0,297)$ (Tabela 1). O animais apresentaram evolução pós-operatória adequada até a data do sacrifício. Não foram observadas complicações da ferida operatória, incluindo infecção ou deiscência, nos quatro subgrupos de animais. A análise estatística do estudo da força de ruptura não demonstrou diferença significativa entre os valores dos quatro subgrupos $(p=0,262)$ (Figura 1). Os resultados da concentração de hidroxiprolina, nos fragmentos da parede abdominal, não evidenciaram diferença estatística quando comparados os quatro subgrupos ( $\mathrm{p}=0,392)$ (Figura 2). 
TABELA 1. Pesos corporais, em gramas (g). Médias e desvios-padrão dos quatro subgrupos de animais, no dia anterior ao procedimento operatório.

\begin{tabular}{l|llll}
\hline Subgrupos & C7 & T7 & C14 & T14 \\
\hline Média & 427,22 & 436,07 & 432,48 & 446,89 \\
\hline Desvio-padrão & 18,39 & 26,76 & 22,31 & 24,74 \\
\hline
\end{tabular}

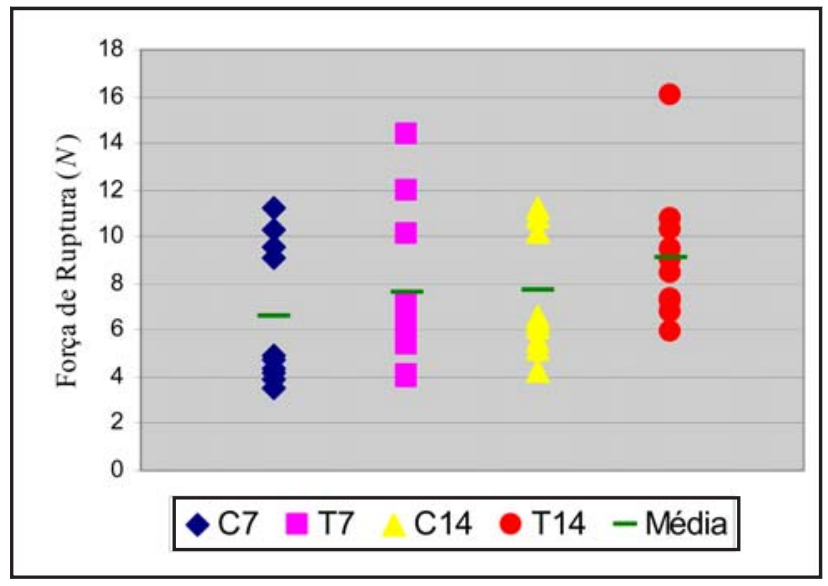

FIGURA 1. Força de ruptura, em Newton $(N)$. Valores e médias dos quatro subgrupos de animais.

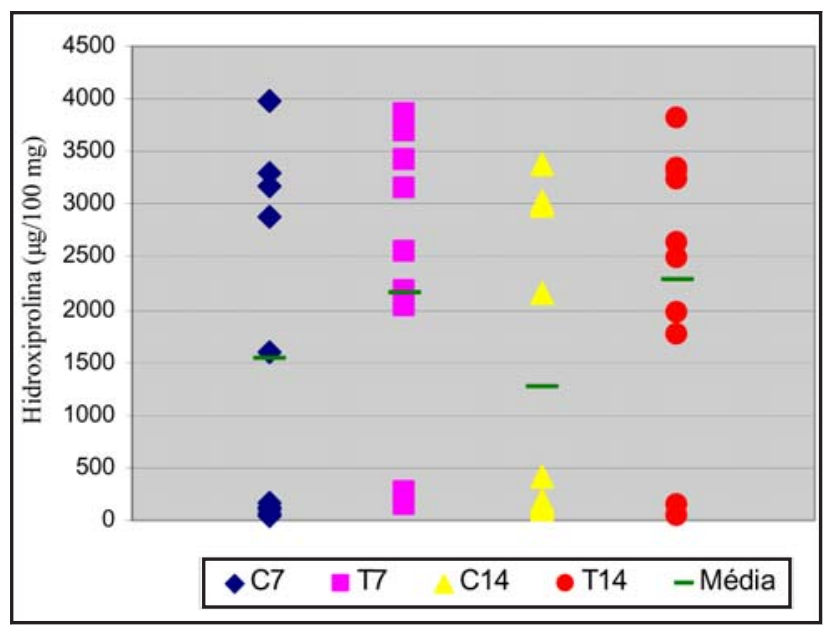

FIGURA 2. Concentração de hidroxiprolina, em $\mu \mathrm{g} /$ $100 \mathrm{mg}$ de tecido seco. Valores e médias dos quatro subgrupos de animais.

\section{Discussão}

O processo de cicatrização envolve uma série de eventos celulares e morfológicos em resposta à lesão tecidual, iniciando-se com hemostasia e inflamação, seguida da regeneração do tecido conjuntivo e da remodelagem da ferida ${ }^{17}$. Portanto, o uso dos antiinflamatórios no pósoperatório, pela eficácia em reduzir a dor e a inflamação, induz a necessidade de avaliar, além das toxidades já reconhecidas no trato gastrintestinal, nos rins e nos outros sistemas orgânicos, os riscos potenciais ao processo de cicatrização das feridas cirúrgicas.

No presente estudo, visto que até a data de sacrifício, $7^{\circ}$ ou $14^{\circ}$ dia de pós-operatório, nenhum animal dos quatro subgrupos apresentou complicação da ferida operatória, tais como infecção ou deiscência, pode-se inferir que o tenoxicam não interferiu no processo de cicatrização da parede abdominal com intensidade suficiente para causar alterações clínicas precoces.

A avaliação da força de ruptura, apesar de expor a cicatriz a situações extremamente adversas, que dificilmente são observadas no pós-operatório, tem sido um parâmetro bastante empregado para avaliar a coesão dos tecidos da ferida cirúrgica nas diferentes fases do processo de cicatrização ${ }^{18,19}$. No presente estudo, como não foi observada diferença estatisticamente significante entre os valores de força de ruptura dos quatro subgrupos, pode-se indicar que o tenoxicam não produziu alterações expressivas no processo de cicatrização da parede abdominal quanto à resistência da cicatriz.

A hidroxiprolina é encontrada quase que exclusivamente como componente do colágeno dos tecidos dos mamíferos, sendo responsável por cerca de $14 \%$ do peso dessa proteína ${ }^{20}$. Portanto, a determinação da concentração de hidroxiprolina pode propiciar uma estimativa do conteúdo de colágeno da amostra tecidual ${ }^{21,22}$. No presente experimento, como não houve diferença estatisticamente significante nos resultados da concentração de hidroxiprolina, nos fragmentos da parede abdominal, entre os quatro subgrupos, pode-se deduzir que o tenoxicam também não produziu alterações expressivas no metabolismo do colágeno.

Resultados semelhantes foram obtidos por Tognini e col. ${ }^{12}$, que também não observaram diferenças significantes nos valores de força de ruptura e na análise histológica da ferida da parede abdominal de ratos sob ação do meloxicam, em comparação ao grupo controle. O meloxicam, pertencente também ao grupo oxicam, se diferencia do tenoxicam por ser levemente seletivo para a ciclooxigenase 2 .

No entanto, os resultados do presente experimento não são concordantes com estudos investigando os efeitos de outros antiinflamatórios não hormonais sobre a cicatrização da parede abdominal. Tognini e col. ${ }^{10}$ e Minossi e col. ${ }^{11}$ verificaram que a cicatriz da parede abdominal de ratos tratados com diclofenaco de sódio apresenta menor densidade de fibras de colágeno no $7^{\circ}$ e $14^{\circ}$ dias de pósoperatório em comparação ao grupo controle. Minossi e col. ${ }^{11}$ observaram ainda complicações como infecção de parede, hérnia incisional e óbito somente no grupo tratado com diclofenaco de sódio. O efeito desfavorável desse antiinflamatório ocorreu também na cicatrização do trato gastrointestinal. De Souza e col. ${ }^{23}$ observaram deiscência de anastomose intestinal, seguida de peritonite e morte, em $20,8 \%$ dos animais do grupo tratado com diclofenaco de sódio, enquanto nenhum caso foi verificado no grupo controle. Por conseguinte, apesar das características similares do tenoxicam e do diclofenaco sódico, principalmente por serem inibidores não seletivos da ciclooxigenase, esses dois antiinflamatórios não hormonais 
têm efeitos diferentes no processo de cicatrização.

Em um estudo para avaliação de agentes antiaderentes sobre a cicatrização intestinal, Uzunköy e col. ${ }^{8}$ verificaram que a administração de uma dose de $1,4 \mathrm{mg}$ de tenoxicam por via intraperitoneal, logo após o término de anastomose cólica, não tinha efeitos adversos sobre o processo de cicatrização do cólon. Os níveis de hidroxiprolina e os valores do teste de resistência à insuflação gasosa não evidenciaram diferenças significantes em comparação ao grupo controle.

\section{Conclusão}

A administração de tenoxicam, por via intramuscular, não interfere na cicatrização da parede abdominal de ratos.

\section{Referências}

1. Makela JT, Kiviniemi H, Juvonen T, Laitinen S. Factors influencing wound dehiscence after midline laparotomy. Am J Surg. 1995;170(4):387-90.

2. Baggish MS, Lee WK. Abdominal wound disruption. Obstet Gynecol. 1975;46(5):530-34.

3. Niggebrugge AH, Hansen BE, Trimbos JB, van de Velde CJ, Zwaveling A. Mechanical factors influencing the incidence of burst abdomen. Eur J Surg. 1995;161(9):655-61.

4. Ferguson MK. The effect of antineoplastic agents on wound healing. Surg Gynecol Obstet. 1982;154(3):421-29.

5. Mather LE. Do the pharmacodynamics of the nonsteroidal anti-inflammatory drugs suggest a role in the management of postoperative pain? Drugs. 1992;44(5):1-12.

6. Vandermeulen EP, Van Aken H, Scholtes JL, Singelyn F, Buelens A, Haazen L. Intravenous administration of tenoxicam $40 \mathrm{mg}$ for post-operative analgesia: a doubleblind, placebo-controlled multicentre study. Eur J Anaesthesiol. 1997;14(3):250-7.

7. Munro FJ, Young SJ, Broome IJ, Robb HM, Wardall GJ. Intravenous tenoxicam for analgesia following laparoscopic cholecystectomy. Anaesth Intensive Care. 1998;26(1):56-60.

8. Uzunköy A, Akinci OF, Coskun A, Aslan O, Kocyigit A. Effects of antiadhesive agents on the healing of intestinal anastomosis. Dis Colon Rectum. 2000;43(3):370-5.

9. Pollack SV. Systemic medications and wound healing. Int J Derm 1982;21:489-96.
10. Tognini JRF, Goldenberg S, Simões MJ, Sauer L, Melo RL, Ortiz PLA. Efeito do diclofenaco de sódio na cicatrização da parede abdominal de ratos. Acta Cir Bras. 1998;13(3):167-71.

11. Minossi JG, Leite CVS, Naresse LE, Rodrigues MAM, Angeleli AYO, Kobayasi S. Efeito do diclofenaco de sódio na cicatrização da parede abdominal de ratos: Estudo histopatológico, da força de ruptura e do colágeno tecidual. Acta Cir Bras. 2001;16(3):146-54.

12. Tognini JRF, Fagundes DJ, Novo NF, Juliano Y. Estudo biomecânico e morfológico da cicatrização da parede abdominal sob ação de meloxicam. Acta Cir Bras. 2000;15(3):146-55.

13. Massone F. Anestesiologia Veterinária. Rio de Janeiro: Guanabara; 1998.

14. Bergman I, Loxley R. Two improved and simplified methods for the spectrophotometric determination of hydroxyproline. Anal Biochem. 1963;35:1961-5.

15. Stegemann H, Stalder K. Determination of hidroxiproline. Clin Chim Acta. 1967;18:267-73.

16. Bussab WO, Morettin PA. Estatística Básica. 4ed. São Paulo: Atual; 1995.

17. Schilling JA. Wound healing. Surg Clin North Am. 1976;56(4):859-74.

18. Scott PG, Chambers M, Johnson BW, Williams HT. Experimental wound healing: increased breaking strength and collagen synthetic activity in abdominal fascial wounds healing with secondary closure of the skin. Br J Surg. 1985;72(10):777-9.

19. Spyrou GE, Naylor IL. The effect of basic fibroblast growth factor on scarring. Br J Plast Surg. 2002;55(4):275-82.

20. Ballantyne GH. Intestinal suturing. Review of the experimental foundations for traditional doctrines. Dis Colon Rectum. 1983;26(12):836-43.

21. Mastboom WJB, Hendriks T, van Elteren P, de Boer HH. The influence of NSAIDs on experimental intestinal anastomoses. Dis Colon Rectum. 1991;34(3):236-43.

22. Minossi JG, Leite CVS, Naresse LE, Rodrigues MAM, Curi PR, Kobayasi S. Ação do diclofenaco de sódio em anastomoses realizadas no intestino delgado de ratos. Acta Cir Bras. 1998;13:37-43.

23. de Sousa JB, Soares EG, Aprilli F. Effects of diclofenac sodium on intestinal anastomotic healing. Experimental study on the small intestine of rabbits. Dis Colon Rectum. 1991;34(7):613-7.
Endereço para correspondência:

André Luís Conde Watanabe

SQN 316 Bloco “A” Apartamento 102

70775-010 Brasília - DF

andre med74@hotmail.com

andreluiswatanabe@yahoo.com.br
Conflito de interesse: nenhum Fonte de financiamento: PIBIC - CNPq/UnB (02/0107)

Recebimento: 26/09/2004

Revisão: 03/11/2004

Aprovação: 19/12/2004

\section{Como citar este artigo:}

Watanabe ALC, Watanabe LM. Efeitos do Tenoxicam sobre a cicatrização da parede abdominal: estudo experimental em ratos. Acta Cir Bras [serial online] 2005 Mar-Abr; 20(2). Disponível em URL: http://www.scielo.br/acb 